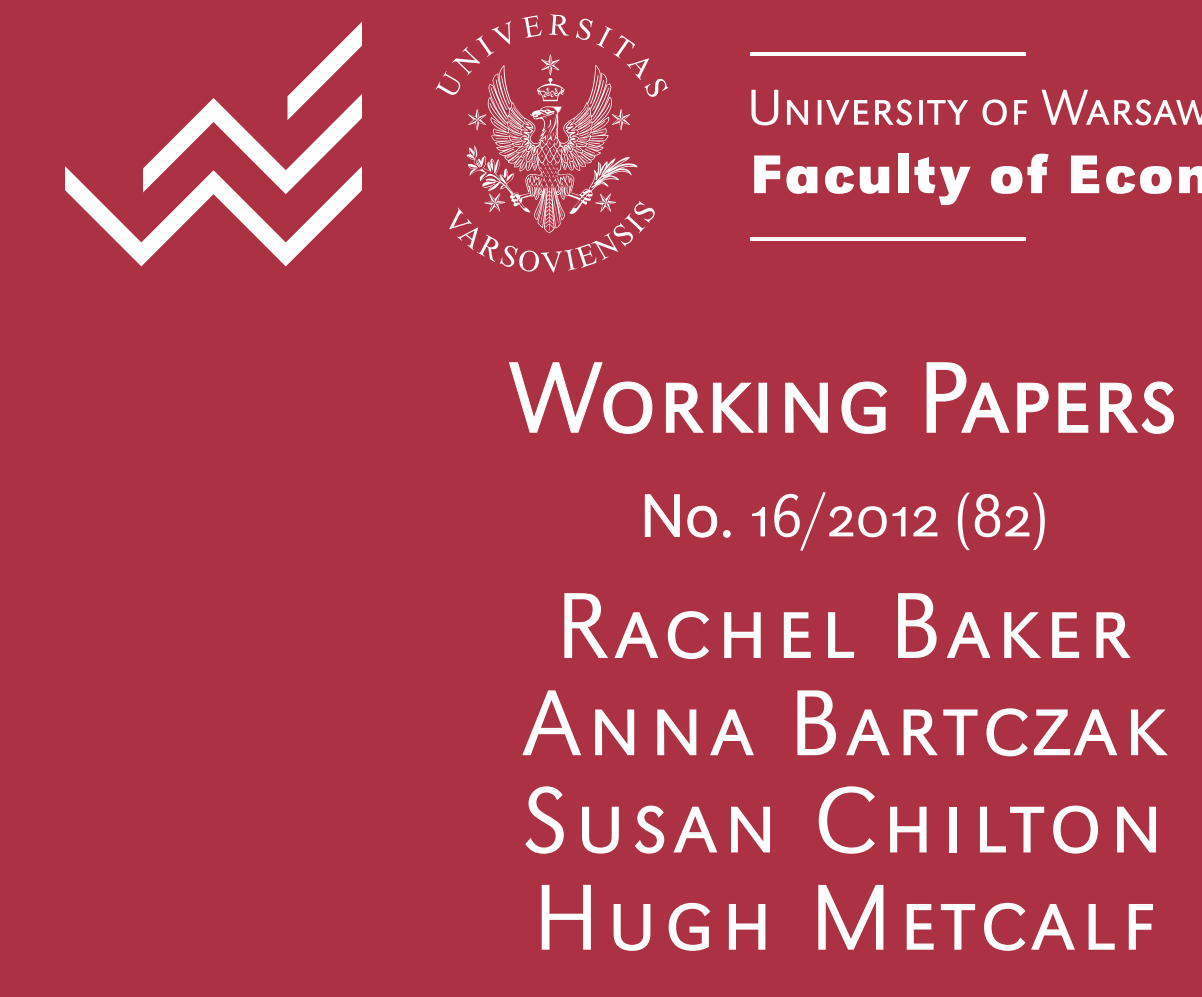

DID PEOPLE "BUY" WHAT WAS "SOLD"? A QUALITATIVE EVALUATION A CONTINGENT VALUATION SURVEY
INFORMATION SET FOR GAINS IN LIFE EXPECTANCY 


\title{
Did people "buy" what was "sold"? A qualitative evaluation a Contingent Valuation survey information set for gains in life expectancy
}

\author{
Rachel Baker \\ Yunus Centre for Social Business and Health \\ Glasgow Caledonian University \\ e-mail: Rachel.Baker@gcu.ac.uk

\section{Susan Chilton} \\ Newcastle University Business School \\ e-mail: susan.chilton@newcastle.ac.uk
}

\author{
Anna Bartczak \\ Warsaw Ecological Economics Center \\ Faculty of Economic Sciences \\ University of Warsaw \\ e-mail: bartczak@wne.uw.edu.pl \\ Hugh Metcalf \\ Newcastle University Business School \\ e-mail: hugh.metcalf@newcastle.ac.uk
}

\begin{abstract}
A number of stated preferences studies have quantified the value of gains in life expectancy from pollution control and use a Value of a Life Year (VOLY) approach to calculate the value placed on avoiding premature mortality following exposure to such pollution. However, life expectancy gains are a complex concept and no attempt has been made, to date, to investigate peoples' understanding of what it is they are being asked to value. This paper uses a structured debriefing exercise to qualitatively investigate an approach which explicitly emphasises how this gain is delivered. We find that, for the majority of respondents, the approach is effective in communicating the ongoing nature of the gain and reduces the use of the (incorrect) heuristic that it is an 'add-on' at the end of life, in poor health. Further refinements are required, however, to communicate the cumulative nature of these risk reductions and the lack of impact on quality of life.
\end{abstract}

\section{Keywords:}

air pollution reduction, contingent valuation, gain in life expectancy, information set and provision, qualitative debriefing survey, quality of life, Value of a Life Year (VOLY)

JEL:

I15, I18, Q51, Q53, Q56 


\section{Introduction}

The monetary value of health benefits or costs to individuals from different environmental regulations has been increasingly requested by government departments in the European Union countries and the USA for use in cost-benefit analyses of these programmes. Desaigues et al. (2011) note that whereas all valuation studies before 1996 calculate the economic cost of mortality as a number of premature deaths due to pollution multiplied by the value of prevented fatalities (also called 'Value of Statistical Life' (VSL)), in recent years there has been a growing recognition that the VOLY (Value of Life Year) measure is the more appropriate approach to deploy when valuing the life expectancy gains arising from reductions in mortality. Advantages of using the VOLY over the VSL approach are discussed in detail in several studies (see e.g. Brunekreef et al (2007), Hammitt (2007) and Desaigues et al. (2011)) but a major factor is that it is more theoretically appropriate, capturing as it does the fact that the gain in life expectancy results from annual, ongoing risk reductions, as opposed to a one-period approach which underpins the VSL (see Section 2 for further discussion). These risk reductions to individuals within the affected population generate the population-based estimate of life expectancy, which expresses how many more months/years an average individual of a particular age can expect to live. So, for example, a 40 year old male in the UK has a life expectancy of 38 years (although some will die before they reach 78 while others will live longer).

Using the VOLY approach requires the adoption of stated preference techniques such as the Contingent Valuation Method (CVM) and, as such, provides us with a vehicle by which to investigate an issue of central importance to the CVM - that of information provision and, specifically, its impact on respondent interpretation and understanding of the benefits (sometimes termed the health or environmental "good") being valued. It is striking that this issue has been largely overlooked in the literature to date. Qualitative investigation alongside CVM studies to date have focussed on understanding the reasons for and/or the validity of WTP responses or exploring the efficacy of focus group techniques as a tool for developing the description of the good for the purposes of questionnaire design (see Baker et al., 2008 for a detailed review). The results of ex post debriefing - a common practice in CVM studies - if reported, rarely provide insights into respondents' interpretation and assimilation of the information with respect to the "good" and what they perceive it to be. This is because it is usually carried out for other purposes and is often informal in nature and specific to the particular study.

If Payne et al.'s (1999) constructivist interpretation of contingent valuation is accepted, then the issue of respondent understanding of the information set is of crucial importance. They argue that a central role of any elicitation procedure is to aid the respondent in arriving at 'well-constructed' preferences and that respondents must give thorough consideration to the most critical information and not be unduly influenced by irrelevant information or features such as survey design characteristics or framing. Considering complex health or environmental goods in CVM studies, there are three approaches to the problem of information provision. One approach, which certainly avoids information overload, is to describe the good in very general terms. In the context of life expectancy, this generally means providing no details as to how the change comes about, instead focussing solely on the outcome for the average person exemplified by the following:

"The chance for a man/woman of your age to become at least 75 years old is $x \%$. On average, a 75-year old lives for another 10 years. Assume that if you survive to the age of 75 years you are given the possibility to undergo a medical treatment. The treatment is expected 
to increase your expected remaining length of life to 11 years. Would you choose to buy this treatment if it costs y and has to be paid for this year?" (Johannesson and Johansson, 1996 and 1997) ${ }^{1}$

"By reducing the general level of air pollution that causes wear and tear and faster ageing, everybody could live longer. That would mean that you (and everyone else in your household) could expect to live about $X$ months longer in your (their) normal ${ }^{2}$ state of health" (Chilton et al. 2004)

Whilst this arguably reduces the cognitive burden on respondents, the cost of this strategy to the validity of any resulting willingness to pay (WTP) estimates of the value of a life year (VOLY) is unknown, but anecdotal evidence from our own previous experience and that of colleagues suggests that many respondents adopt the heuristic that it is a simple 'add-on' at the end of life, most usually in poor health and value this accordingly, as opposed to what is actually delivered (changes in the risk of death over time).

A second approach, never tried for obvious reasons, would be to describe it in very precise, technical terms, perhaps based explicitly around equation 2 in Section 2. A third approach, which in fact mirrors standard practice in most contemporary CVM surveys, would be to provide fairly detailed information to respondents, checking for cognition and comprehension problems during the survey development phase, either within focus groups or through individual verbal protocols or a combination of both. However, there has been little rigorous research exploring the provision of information on the detailed understanding of a complex good in a CVM study. In this paper we explore the issue of information provision qualitatively in an in-depth, structured debriefing exercise focused on the "good" being valued.

We suggest that a necessary condition for such an information set is that it places respondents in a position whereby they are "buying" what the survey is "selling", as opposed to their own (possibly erroneous or embellished) perception of the good. Put another way, a precursor to a "successful" or more accurate valuation is that respondents have at least a sound intuitive understanding of the goods main characteristics and how changes in its level of provision might affect their wellbeing. Such an outcome is crucial if respondents are to be able to construct more reliable and stable preferences over the good which, in turn, should (positively) affect the reliability of the resulting valuations.

The starting point for our investigation is the results from two previous UK VOLY studies (Desvousges et al., 2007 in which aggregate - EU - results are also reported in Desvousges et al. 2011 and Chilton et al. 2011). In both studies, carried out on a convenience sample of members of the public in Newcastle upon Tyne, sample size was identical (152) and demographic characteristics very similar. The only major difference between those studies was the nature and provision of the information set presented. Although both were based on the same pictorial/graphical depictions of life expectancy changes, the first study employed quite brief (standard) supplementary information. In the second study (Chilton et al. 2011) more effort and time was devoted to delivering and facilitating understanding of the nature of the good in question by respondents, resulting in a longer value construction phase. This included a verification of respondents' understanding of a life expectancy gain prior to the

\footnotetext{
${ }^{1}$ As far as we are aware, the 1996 survey was the first to ask explicitly about the valuation of a life expectancy gain.

${ }^{2}$ Respondents had previously discussed what "normal" state of health mean for them in different stages of their lives.
} 
valuation. The results of those two surveys differ significantly suggesting that respondents in the two surveys were "buying" different goods.

Two characteristics that are particularly indicative of underlying reliability of WTP are the degree of scope sensitivity and the prevalence of true zero bids. Thus, it is expected that WTP might be significantly higher - but not necessarily double, given diminishing marginal utility - for the doubling of the offered life expectancy gain ( 3 months to 6 months). It is also not unreasonable to expect that more or less the same proportion of respondents would have little or no interest in the good and/or prefer to spend their limited budget on other things in each study, since the policy deliverable - life expectancy gains - had not changed. In the first of these studies, the response set was characterised by a low degree of scope sensitivity (1.4, meaning that a 6 month life expectancy gain only generated a $40 \%$ increase in WTP over the 3 month gain) and a low percentage of true zero bids (7\% for the 3 month gain). This contrasts with the second study in which the comparable figures were, respectively, 2.2 and $26 \%$. While the difference in results (WTP profiles) is clear, this does not constitute definitive proof that a better respondent understanding was present in the second study. Other (unidentified) differences may have been at play. This is important, since the process is labour intensive and cognitively more demanding for respondents so it is important to verify that it is indeed worth it and also, if possible, to draw out some general and context specific lessons for future studies.

Returning to VOLY elicitation, generally, one of two approaches are adopted: either to ask respondents to directly value a specific life expectancy gain or, alternatively, to value a reduction in their risk of dying over an $\mathrm{N}$ year period (Morris and Hammitt, 2001; Krupnick et al., 2002; Alberini et al., 2004). Both are in fact equivalent (Hammit, 2007), the latter simply values life expectancy changes indirectly (technically defined in Section 2). But this is never explained to respondents. Theoretically speaking this should not matter and, as long as the provision mechanism is not of interest to the first set of respondents nor the outcome of interest to the second set of respondents, it should not matter from a preference construction/valuation perspective either. The information set from Chilton et al. (2011) in fact allows respondents access to both pieces of information, in case this is not the case. We focus (for the rest of the paper) on the information set provided to respondents in that study and its examination through a systematic and in-depth debriefing, qualitative study. We outline the aims and objectives of our qualitative debriefing study in Section 3 below, immediately prior to discussing its results (Section 4) but our assessment (Section 5) will show that the approach adopted was successful in reducing significantly the use of the 'add on' heuristic above, which should positively impact the reliability of WTP responses but further information of better presentation of the cumulative nature of the gain will be required in future studies. It also appears that some attributes will remain ill-defined in respondents' minds and we suggest solutions to this problem in Section 5.

We commence by formally defining a change in life expectancy (Section 2).

\section{Defining a change in life expectancy}

\subsection{The epidemiological-based definition}

Changes in average life expectancy are generated by changes in existing hazard rates (risks of death) to individuals making up that population over their lifetime - hence the emphasis on valuing individual risk reductions in some VOLY studies to date, as noted earlier. It is possible to specify more formally this intuition in that life expectancy (LE) (eqn. (1)) is given 
by the area under a survival function, $S(t)$, given the probability that individual survives until at least time t:

$$
L E=\int_{0}^{\infty} S(t) d t,
$$

The general properties of the mortality and survival functions are well-established (see, for example, Jenkins (2005)) and will not be expanded on here. The key point is that changes in the hazards, or risks, faced by an individual over her/his lifetime changes the area under the survival function i.e. changes life expectancy. Notably, a specific gain in life expectancy can be generated as a result of an infinite number of different perturbations in the hazard function but, for illustrative purposes, eqn. (2) presents the basic definition of remaining life expectancy, LE, in discrete time, for a 40-year-old, expressed for convenience in decades, in which $p_{\mathrm{i}}$ represents the existing hazard rate for a given decade:

$$
L E=10\left(1-p_{40}\right)+10\left(1-p_{40}\right)\left(1-p_{50}\right)+10\left(1-p_{40}\right)\left(1-p_{50}\right)\left(1-p_{60}\right)+. .
$$

with $p_{90}=1$, implying that for the purposes of this exposition, all people will die not later than age 90. In turn, eqn. 3 reflects the change in life expectancy derived from a programme such as particulate air pollution reduction ( $\left.\mathrm{LE}_{\mathrm{PR}}\right)$, in which the hazard rate reduction, $k(k \in(-1,0))$ is a constant and proportional to the initial level of the hazard rate (as evidence in Pope et al. 1995 suggests is the case):

$$
L E_{P R}=10\left(1-p_{40}(1+k)\right)+10\left(1-p_{40}(1+k)\right)\left(1-p_{50}(1+k)\right)+. ., k \in(-1,0)
$$

As noted earlier, it is not surprising that CVM practitioners do not present information to survey respondents in this manner. The challenge is to communicate it in a manner that could be understood and assimilated by the average respondent. We now describe the key features of the alternative method, along with a brief description of associated procedures ${ }^{3}$.

\subsection{The CVM survey definition (Chilton et al., 2011)}

It is traditional practice within most CVM studies to present the information set - be it textual or pictorial - in a 'block' followed by the WTP question(s). Within the Chilton et al. (2011) study the information was presented in a small group setting via a combination of interactive discussion, pictorial diagrams describing how a life expectancy gain is derived and some structured questions to check for comprehension and misunderstanding. We repeated the same procedures for the purposes of the qualitative study reported in this paper ${ }^{4}$, and followed the presentation of information with two WTP questions for one and six month life expectancy gains. This served as a starting point for individual-based qualitative debriefing interviews, which immediately followed the group sessions and which form the basis for our qualitative analysis.

\footnotetext{
${ }^{3}$ Whilst the CVM survey is not the focus of this paper, it is available on request and may be helpful to those interested in the full information set - space considerations preclude reproduction of the details of that survey here.

${ }^{4}$ As such, we chose not to collect qualitative information during the information processing phase, since we were not exploring the value construction process per se. In this study, we focus rather on the outcome of this process.
} 
Here, we outline only the main components of the information set. Firstly, participants were given some basic information regarding the health effects of air pollution, including our increased vulnerability as we age and there was some discussion about perceived air quality in their neighbourhood. They were then introduced to the diagrams, along with clear, verbal explanations of what they signified. These diagrams were based around a so-called 'ability to survive' curve which depicts the physical ability of the body to survive as a person ages. It simply reflects the intuition (and fact) that as people age, they become more susceptible to illnesses and accidents until at some point we can no longer expect to survive. Figure 1 shows such a curve for an average 20 year old 5 .

Figure 1. 'Ability to Survive’ Curve (Average 20 Year Old)

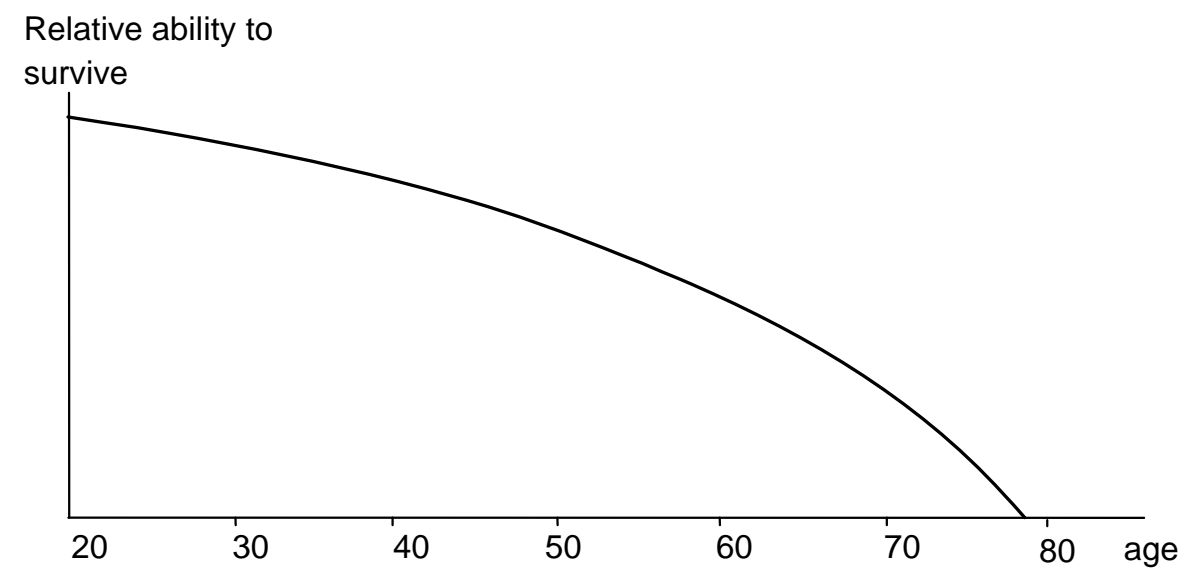

This was followed by an explanation of how a reduction in air pollution impacts the curve i.e. shifts it outwards and upwards, increasing the expected age of death (Figure 2). It was emphasised that the smaller the risk reduction the smaller the shift. To the extent that this pictorial representation displays an increasing vertical gap between the original and the post pollution reduction 'ability to survive' curves and hence indirectly the original and the post pollution reduction survival curves- which is precisely what would result from an ongoing proportionate reduction in the hazard rate.

\footnotetext{
5 Individual 'ability to survive' curve diagrams were provided to respondents according to their own age group.
} 


\section{Figure 2. 'Ability to Survive’ Curves Following Different Reductions in Air Pollution.}

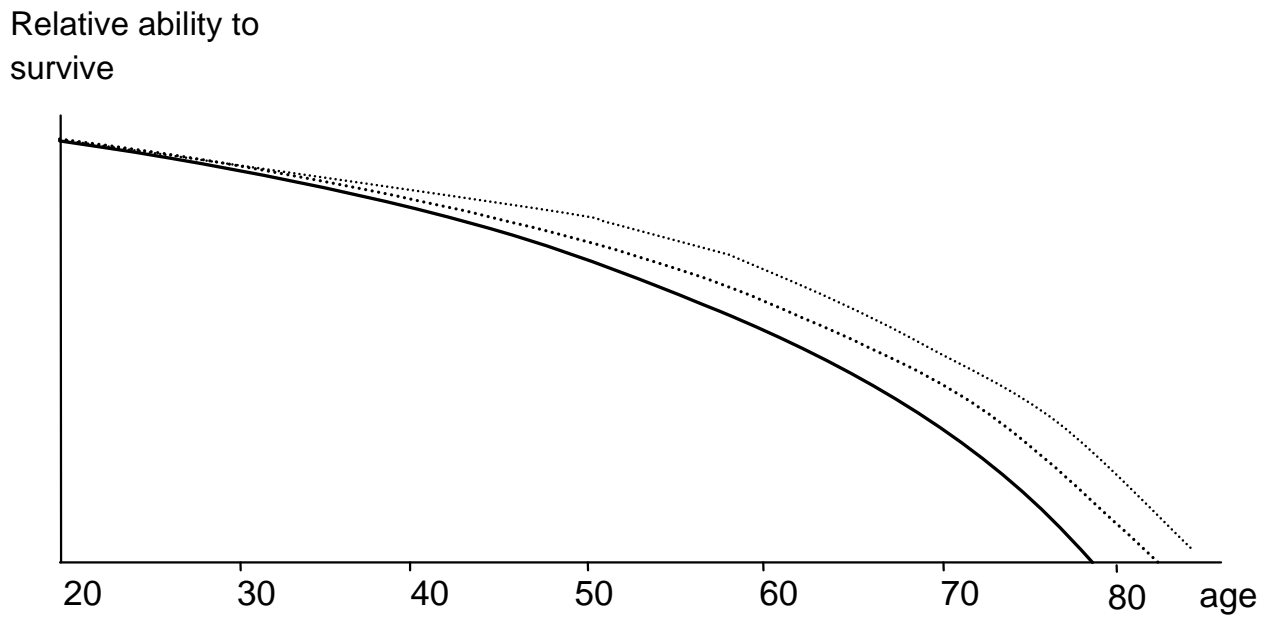

To emphasise the cumulative (ongoing) and increasing nature of the gain, it was stressed that while most of the risk reduction and hence the effect on life expectancy, occurs towards the end of a person's life, conditional on them reaching this stage, some benefit accrues immediately the risk reduction is implemented ${ }^{6}$. Figure 3 served as an 'en-route quiz' to check how respondents were assimilating the information so far, although they had been encouraged to ask questions and/or seek clarification as they went along if they wished to. They were asked what would happen to air pollution levels and hence the 'Ability to Survive' curve if the air pollution policy ceased at point A and current levels of air pollution resumed (the correct answer is that it would approach but not coincide with original life expectancy). Initially, some respondents answered this correctly while others did not. Further clarification was provided as necessary, providing a vehicle by which to ensure all respondents were aware of the correct answer .

\section{Figure 3. 'Ability to Survive' Curves Following a Return to the Original level of Air Pollution}

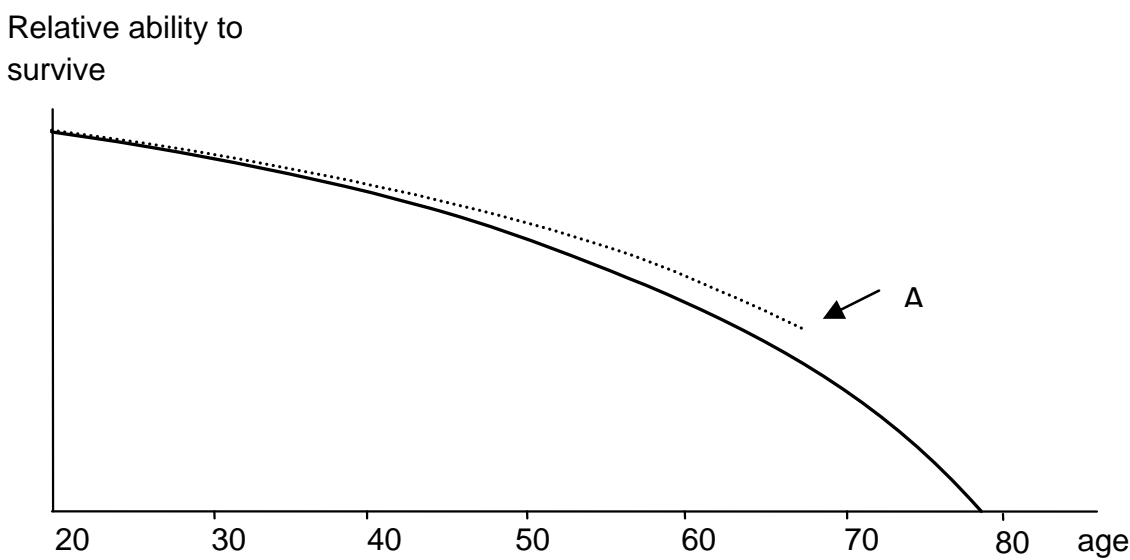

\footnotetext{
${ }^{6}$ It was also pointed out that the change in expected age of death was magnified for illustrative purposes and did not constitute the addition of a substantial number of years at the end of life.
} 
Whilst it is the case that policymakers only have evidence on average life expectancy ${ }^{7}$ it is possible that an individual's WTP may depend on how they perceive themselves relative to the average, in particular in terms of their health, genetics and other circumstances. Depending on this, it might be the case that the (perceived or actual) shift in their personal 'ability to survive' curve would differ significantly more or less than the average shift. Therefore, participants were asked to rate their own health state (now and over time) to that of an average person. Information was provided on how any air pollution reduction might affect people of average, below average and above average health in order to highlight the uncertainties that surround an individual's gain in their own life expectancy. Thus, depending upon their health, genetic make-up and general lifestyle and lots of other things, including where they live, the effect of air pollution may have a much greater or much smaller impact on them individually. Uncertainty was emphasised to counteract the heuristic that the life expectancy gain was a 'certain' addition to life at the end. Chilton et al. (2011) report that explicit attention was not drawn to irrelevant (to policymakers, at least) benefits such as impact on quality of life or the environment as in earlier piloting it seemed to highlight these features to some respondents that may otherwise not have considered it. We therefore followed to ensure that the information set remained constant across their study and our study.

Taken as a whole, the information set presentation lasted approximately 35 minutes, after which WTP values were elicited. Participants then proceeded to the qualitative interview.

\section{The Qualitative Debriefing Study}

When CVM respondents provide a WTP value they are being asked to express, in money terms, the value of the externality - in this case changes in life expectancy - provided by policy (here, air pollution reduction). If the resulting value is to be used in a cost-benefit analysis of only this policy deliverable - with other benefits such as reduced levels of illness or environmental benefits valued separately, or not at all, as is usually the case - then elicited values should not be augmented by consideration of other impacts such as, for example, in the case of air pollution reduction, consideration of the impact on others, environmental benefits or impacts on quality of life. In the spirit of Payne et al. (1999) these are effectively irrelevant and should not influence responses. Nevertheless, a combination of evidence and experience suggests that at least some respondents may be incorporating irrelevant information in their valuation of life expectancy gains. In order to draw any general (or context specific) lessons for CVM, it is first necessary to identify whether our respondents augment their values with irrelevant information,

Therefore, there were two distinct aims to this qualitative investigation. The first was to ensure that respondents understood the key characteristics of the 'good' as defined by us, in particular the cumulative and uncertain nature of the gain i.e. that the gain in life expectancy for each individual is uncertain and is a result of cumulative (i.e. ongoing and increasing) changes in their chances of survival over their lifetime as opposed to an 'add-on' at the end of life and, further that the reductions in risk of death begin almost immediately on inception of the policy, but with most accruing later in life. The second was to assess the impact, if any, of irrelevant factors on their perception of the good and its subsequent valuation. In order to facilitate these aims, we devised a structured debriefing interview based around a set of cards,

\footnotetext{
${ }^{7}$ And hence would use the average in any calculations in of aggregate life expectancy gains.
} 
followed by some open-ended questions, described below, focusing on respondent comprehension and understanding 8 .

\subsection{Procedures}

Debriefing within CVM studies can take a number of forms, ranging from structured/ closed ended (specific questions at the end of the survey to be answered and recorded) to more an open ended, qualitative approaches, including probing by the interviewer to check for misunderstanding, WTP reliability and so forth. Within our debriefing exercise while we include an exploration of the WTP responses, the primary purpose of which is to serve as a medium with which to (indirectly) explore respondent understanding of the good. Further questions were designed to try and access this issue more directly ${ }^{9}$.

Interviewers (study authors) used a semi-structured interview schedule that began with a warm up question asking for a very general response to the information set, following which respondents were asked to complete a simple 'card-sort' procedure about the factors they took into account in formulating their WTP values, the main function of which was to stimulate and structure the discussion. Respondents were asked to arrange the cards into three piles corresponding to whether they had made use of each factor in when constructing their WTP $^{10}$. The three piles were labelled "Used this Factor", "Did not use this factor" and "Did not use but seems to matter now" and their arrangement of the cards was used as a natural prompt for open ended discussion.

There were 13 cards, each listing a separate factor. These factors were defined by us and comprised a mix of actual characteristics of the good and extra (so-called irrelevant) factors which we knew were likely (from previous CVM studies, both of air pollution reduction and more generally) to be considered important to respondents during the value construction process, but which were irrelevant to the policy deliverable.

Five cards described attributes which are scientifically important to the delivery of a life expectancy gain. These cards were: "my increased ability to survive" (reflecting the cumulative nature of the gain and the reduced risk of dying); "my health and genes" reflecting a major factor in underlying uncertainty in respect of any individual gain); "number of months" (length of expected gain); "my budget"; and "paying for the rest of my life" (reflecting the need for on-going payment if the full life expectancy gain is to be realised).

The remaining eight cards described factors such as: "effects on other people"; "my quality of life"; and "environmental issues" which, if chosen as important factors by respondents, could have led to them to embellish the good in some way ${ }^{11}$, potentially inflating its value. In addition, selecting the card "whether air pollution policy will work" indicated a respondent brought their own concerns to the exercise, in this case scepticism towards the scenario presented to them. The rest of the cards were not relevant to understanding life expectancy

\footnotetext{
${ }^{8}$ This mirrors the practice adopted in studies such as Baker and Robinson (2004). These researchers employ a range of methods from schools of qualitative thought, chosen to suit the particular research objectives.

${ }^{9}$ The full debriefing qualitative interview script is available on request.

${ }^{10}$ Although some of these cards would be considered 'valid' and others 'problematic', no signal was sent out regarding the researcher's views on the correctness of each card.

${ }^{11}$ Embellishment of the good is not a new phenomenon and is not confined to life expectancy gains and is almost certainly present in many valuation studies. Chilton and Hutchinson (1999) discuss the concept in detail and highlight the role that focus groups can play in determining both its presence and influence.
} 
gains but were issues identified in earlier piloting as important to at least some respondents. These cards are: "other information I have heard about air pollution"; "the level of air pollution where I live"; "my general luck in life"; "my risk of dying from air pollution". In extremis, such considerations could form the basis for the entire valuation ${ }^{12}$.

Once sorted, respondents were asked to take the cards that they had indicated were relevant to their valuations and rank order them according to importance. Using the participants cardsort as a starting point, the interviewer then asked participants to explain why they were important and how they influenced their value construction. General probes were:

- "Why was it important?"; "In what way did that matter to you?"; "What were you thinking about here?”

Respondents were also asked say a little about any key attributes they 'did not use' to investigate reasons why they were not important and to confirm that they understood the cards.

The card-sort was followed by a small number of open-ended questions and the interview concluded with a small number of 'debriefing questions' relating to various aspects of the exercise, the most central to this paper being two questions designed to explore whether respondents actually 'bought' the 'good' that we 'sold' - the essence of a contingent valuation. If they did, then this is a necessary - but not of course not sufficient - condition for a reliable valuation.

- So you said you would pay $£ / P L N X$ for say a 6 month gain in life expectancy. If you were to explain to a friend what you had 'paid for', how best would you describe it to them?

And at the end of the interview:

- Some people have described the gain in life expectancy as an additional 6 months at the end of their life. Would you agree with that?

\section{o Why? Why not?}

The interview schedule formed the common core of all interviews, and was designed to generate the type of qualitative data necessary to answer our specific research objectives but was treated as a flexible research tool so that new themes or issues could be accommodated and explored if they arose.

\subsection{Sample}

The main study was preceded by 12 pilot interviews, 6 in each country ${ }^{13}$. The purpose of the pilot interviews was to refine the interview schedule based on the comprehension and comments given by the pilot respondents and to identify the nature of the data we might expect to collect.

\footnotetext{
${ }^{12}$ Whilst it could be argued that that this value would have some validity to the individual concerned, it is less clear that it would be ethically defensible to use them in resource allocation decisions involving public money. ${ }^{13}$ The final pilot group in the UK included only two respondents and as no changes were made to the qualitative interview script used in the subsequent main study and hence they were retained in the main study.
} 
The main study sample consisted of 49 interviews administered with 24 British and 25 Polish citizens. Respondents were recruited by a market researcher in Newcastle upon Tyne in the UK and Warsaw in Poland. There is some evidence in the literature to suggest that demographic characteristics such as gender and age may impact how respondents react to such information and assimilate it to provide a valuation (e.g. Avitia et al., 2011; Nielsena et al., 2003) while other studies suggest such characteristics do not matter (e.g. Krupnick et al., 2000; Sundström and Andersson, 2009). Blomquist et al. (2011) find evidence in support of both positions, in respect of age. A priori, we had no evidence from previous studies regarding information assimilation when valuing life expectancy gains so, while our sample is not in any way representative, we tried to ensures a reasonable variation in such factors should they matter. A range of income levels were included and just under half of each sample were under 40 . Women were slightly overrepresented in each case $(55 \%$ in Poland and $63 \%$ in the UK).

\subsection{Data analysis}

The interviews were audio recorded and transcribed verbatim. For the purpose of data analysis transcripts were imported into NVIVO qualitative software (QSR 2007) and a series of participant attributes were associated with each transcript (age, gender, WTP values, and country). Analysis followed standard qualitative procedures (see, for example: Mason, 1996; Silverman 2001). The first stage was familiarisation with the transcripts and involved reading and re-reading and listening to audio recordings. Next, qualitative data were categorized using initially broad coding categories before refining and re-defining categories and introducing subcodes. Early coding was carried out by all team members and was based on an initial subsample of transcripts which was used to derive a common coding frame. Each transcript was then coded by one of the authors and a small subsample (6) was coded by two authors to ensure consistency. An iterative process followed, including an ongoing discussion of emergent themes at regular meetings of the authors to ensure that the coding scheme was both relevant to the research questions and grounded in the data. Coded text was examined and interpreted through similar discussions between authors. These steps were iterative, repeated and overlapping rather than discrete, consecutive tasks. Constant comparison techniques, derived from grounded theory (Glaser \& Strauss, 1967) were employed to critically consider thematic categories.

\section{Results}

Results, reported here, are based on the qualitative data from the interviews described above. On average each individual interview took around 20 minutes. For expositional purposes, we report the results of the card-sort separately, although data from both parts of the interview both were used simultaneously in our overall assessment of how respondents reacted to our information set. Table 1 contains an 'overview' of how each factor (card) was treated by respondents in the sense of whether it influenced their valuation, although analysis of the transcripts provides richer insights as will be shown below. In general terms, selecting the five cards containing characteristics might be viewed more favourable, while selecting the irrelevant factors might be viewed as less favourable. Indeed, at this most basic level, key factors ${ }^{14}$ featured more prominently in people's minds than exogenous or contextual effects,

\footnotetext{
${ }^{14}$ Two cards (my budget' [card 1] and 'paying for the rest of my life [card 5]) are key aspect s of the valuation exercise - as opposed to the life expectancy gain itself - and will not be considered further in this paper.
} 
with one exception - 'quality of life' (card 10) providing us with the first indication that the most important irrelevant factor affecting respondents' valuation was 'quality of life'. This initial impression was further verified in the analysis of the data as a whole.

Table 1. Percentage of respondents indicating each attribute as an important influence on their WTP

Cards

\begin{tabular}{|c|c|c|}
\hline & British & Polish \\
\hline $1^{*}$ - my budget & 96 & 80 \\
\hline $2 *$ - my increased ability to survive & 52 & 58 \\
\hline $3 *$ - my health and genes & 74 & 68 \\
\hline $4^{*}$ - number of months & 79 & 58 \\
\hline $5^{*}$ - paying for the rest of my life & 66 & 51 \\
\hline 6 - my risk of dying from air pollution & 48 & 20 \\
\hline 7 - my general luck in life & 9 & 13 \\
\hline 8 - the environment & 37 & 31 \\
\hline 9 - whether air pollution policy will work & 39 & 37 \\
\hline 10 - my quality of life & 70 & 66 \\
\hline 11 - the level of air pollution where I live & 30 & 60 \\
\hline 12 - other information I have about air pollution & 18 & 28 \\
\hline 13 - effects on other people & 26 & 47 \\
\hline
\end{tabular}

In the section that follows we describe the central themes emerging from the data in both the UK and Poland. Quotes illustrating our findings are identified by a respondent's age, gender, stated WTP amounts and country.

In the section that follows we describe the central themes emerging from the data in both the UK and Poland. Quotes illustrating our findings are identified by a respondent's age, gender, stated WTP amounts and country.

\subsection{Cumulative and uncertain nature of the gain in life expectancy}

There is evidence in the coded interview transcripts that the cumulative nature of the gain in life expectancy had been partially understood. Respondents' comments revealed an appreciation of the continuous nature of the gain as opposed to describing it as an add-on of life at the end. Of course, it was rarely described in exactly those terms in respondents' accounts. This is unsurprising since, although the notion was described to respondents using visual aids and examples as described above, we avoided technical terms. Examples of quotes which we judge as indicative of such understanding are presented in Table 2. 
Table 2. Quotes illustrating understanding of the continuous lifetime effect.

\begin{tabular}{|c|c|}
\hline Perception & Quotes \\
\hline \multirow{11}{*}{$\begin{array}{l}\text { Continuous gain over lifetime } \\
\text { as opposed to an 'add-on' of } \\
\text { life at the end }\end{array}$} & "I know it would be stretched out over the time" \\
\hline & 1 month $=£ 25 ; 6$ months $=£ 100 ;$ female; 48 \\
\hline & $\underline{(\mathrm{UK})}$ \\
\hline & $\begin{array}{l}\text { "It's a gradual decline in my health that would } \\
\text { catch up with me at the point of } 72 \text {. Therefore, } \\
\text { those gradual benefits I've gained throughout } \\
\text { the years of my life, it has compounded and } \\
\text { added onto the end." }\end{array}$ \\
\hline & 1 month $=£ 0 ; 6$ months $=£ 50$; male; 33 (UK) \\
\hline & $\begin{array}{l}\text { "I was simply thinking that it increases my } \\
\text { ability to survive in general, that each day } \\
\text { somehow I have a chance for a better life, and } \\
\text { let's say each month we could collect from that } \\
\text { up until } 6 \text { months (gain)." }\end{array}$ \\
\hline & 1 month $=300$ PLN; 6 months $=600$ PLN; \\
\hline & female; 29 (PL) \\
\hline & $\begin{array}{l}\text { "It means when I was thinking about that, I } \\
\text { was thinking that at the very end of my life I } \\
\text { would have these } 6 \text { additional months. But I } \\
\text { think, that if I talk about that with someone, I } \\
\text { suspect, that I would try to show that he/she } \\
\text { would get those days every year, somehow } \\
\text { longer. Summing up, we do not know if we will } \\
\text { live till } 60 \text {, } 80 \text { or } 30 \text {. But it's beneficial for a } 40 \\
\text { year old as well - he/she would get something } \\
\text { from it." }\end{array}$ \\
\hline & 1 month $=60$ PLN $; 6$ months $=300 ;$ female $; 21$ \\
\hline & $(\mathrm{PL})$ \\
\hline
\end{tabular}

However, while these respondents certainly seemed to notice the gain was not at the end of life, there is little explicit evidence that they had taken on board the fact that the distribution of this gain is not constant but is in fact increasing, although there is some evidence elsewhere in the transcripts that a small minority of respondents - notably in Poland - grasped this fact (see Table 3). 
Table 3. Quotes illustrating the increasing nature of the life expectancy gain.

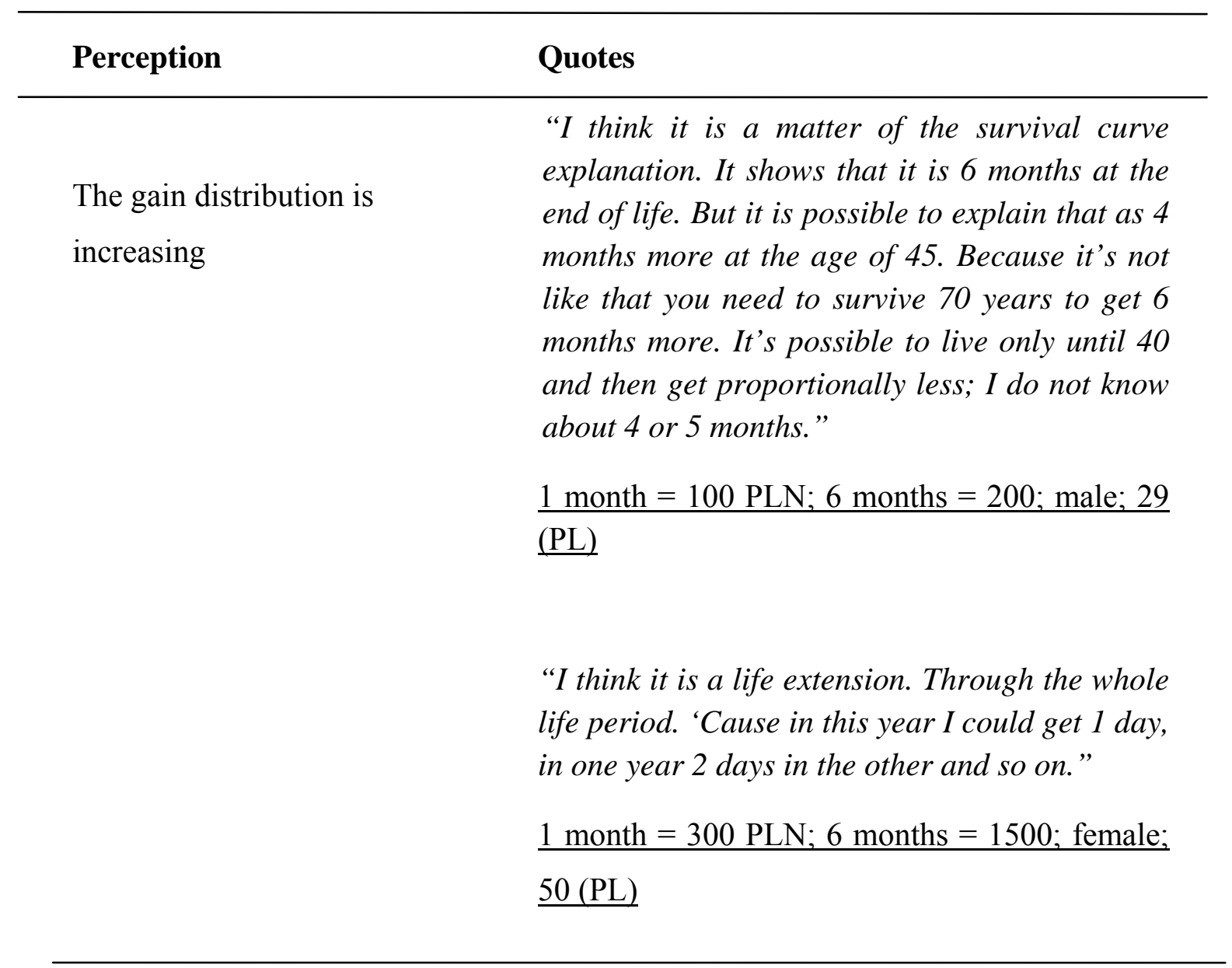

The uncertainty of the gain is another key element that respondents must grasp if the protocol is to be judged as successful in generating understanding. Uncertainty was in fact mentioned by respondents with respect to two different issues (Table 4): the uncertainty of life expectancy and uncertainty concerning the length of a described gain, both of which are appropriate. A reasonable number of respondents compared themselves to the average person in their age group and noted that in their case the length of the life expectancy gain could differ. Some respondents underlined the fact that life expectancy in itself is uncertain, particularly in Poland, where external risks such as car accidents, crime or fate were often alluded to. 
Table 4. Example quotes: the uncertainty of receiving a gain in life expectancy

\begin{tabular}{ll}
\hline Perception & Quotes \\
\hline & "Again, I thought 6 months was worth having, but there again, I \\
said that that perhaps for somebody who was below average \\
[health] they, although they may gain the average, it may be less \\
than that, so I was thinking there was enough uncertainty for me \\
with one month that I was thinking, well, it could be a couple of \\
weeks and you know the actual variability between people is wide \\
the length of LE $\begin{array}{l}1 \text { month }=£ 25,6 \text { months }=£ 100 ; \text { male; } 45 \text { (UK) } \\
\text { gain }\end{array}$
\end{tabular}

"It could be longer life but not necessarily about half a year. It could be one year, but it could also be three months."

$\underline{1 \text { month }=12.5 \text { PLN; } 6 \text { months }=25 \mathrm{PLN} \text {; female; } 26(\mathrm{PL})}$

Uncertainty of

LE in general

"If I am not going to die in a car accident when I am 40, that... in theory..., so I will die, it is a fact... but life quality from when I am 20 till 40 would be better, I would have less illness, at each stage of my life."

1 month $=$ PLN $; 6$ months $=($ PLN $)$ 


\subsection{Quality of life}

Less than half of respondents stated that concerns such as "the environment" (card 8) and "effects on other people"15 (card 13) affected their valuation (see Table 3). However, as noted, our main concern here is the impact on their understanding of the good and, in this respect only one of these factors clearly stands out - quality of life. In both samples, a number of respondents did not disentangle this from the gain in life expectancy and clearly indicated that they considered it to be an integral component of the good, described in terms of how a reduction in air pollution might affect health or quality and/or likelihood of outdoor exercise. In some respondents' minds, the gain in life expectancy would arise because quality of life/health would be improved throughout their life by air pollution reduction. For other respondents - particularly in Poland - quality of life seemed to be an additional and sometimes more important benefit than the gain in life expectancy. Table 5 contains quotes reflecting these features.

\footnotetext{
${ }^{15}$ While almost $50 \%$ of the Polish sample considered this card important, when probed during interview, the majority said that excluding this factor from a valuation process would not change their stated WTP.
} 
Table 5. Quotes illustrating perceived improvement in quality of life.

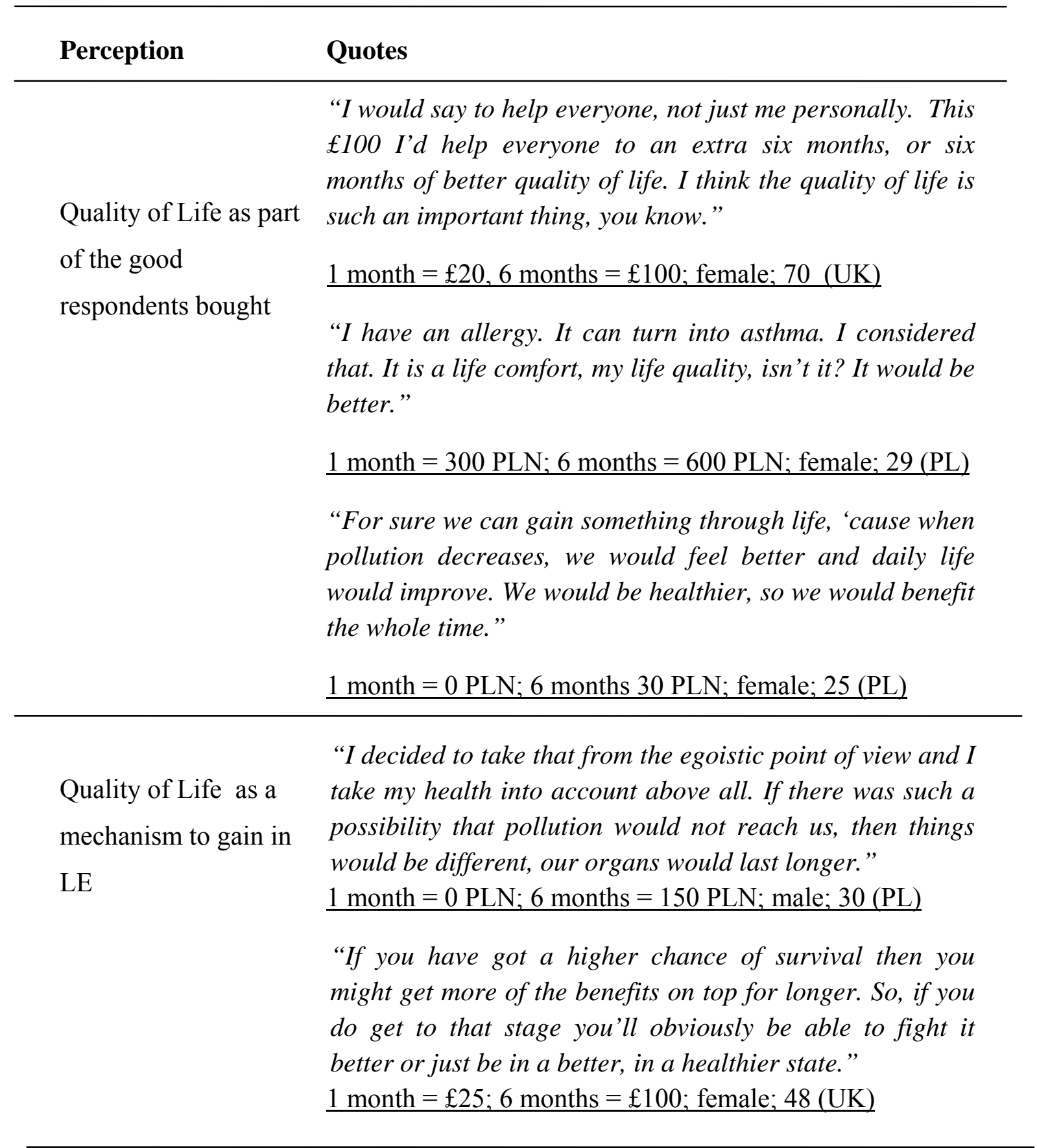

\section{Discussion and conclusions}

To summarise the results from the qualitative study, most respondents appeared to understand the continuous nature of the life expectancy gain and that the gain was in some way uncertain. This constitutes an important step forward and means that such respondents are not constructing their WTP responses for extra months/years at the end of life. However, only some of these same respondents verbalized an explicit understanding that this gain increased over life as opposed to a constant gain each year, implying more information/explanation is required on this aspect. We have not observed systematic 
differences in understanding of the life expectancy gain across samples from Poland and the UK in this survey. So the use of the information set is not, in this case, country/culturespecific. In a broader sense, such a finding might have implications for the increasingly common practice of benefits transfer (Navrud and Ready, 2007), whereby survey values from one site or sample is 'transferred' econometrically to another, either within or across countries.

As noted, many respondents included quality of life improvements, apparently 'adding in' the value they attached to this factor when stating their WTP. There are two potential reasons for this. First, the concept of quality of life is a more familiar and concrete entity compared with shifts in an abstract 'ability to survive' curve and the default heuristic may reasonably be to continue to focus on something they understand. Second, the general description in the CV studies in Desaigues et al. (2011) and Chilton et al. (2011) - and, by necessity, our qualitative study - of the chronic effects of air pollution on the body contained the phrase "we are slightly healthier at each age". We now consider the implications of these findings more generally, both for future CVM VOLY studies and the CVM more broadly. The implications of our qualitative findings for future econometric analysis of quantitative data are to highlight the need for scalar variables to capture or control for the influence of quality of life. It seems clear that careful descriptions of the context in which life expectancy gains are achieved help people to understand the good, but the price the analyst pays is the potential for additional, irrelevant factors to be brought to bear in respondents' valuations. Our conclusion is that this trade-off must be made, and that at least at this stage it is better to try to measure its impact than to try and reduce its impact a priori. Particularly in the case of complex goods where respondents may well be asked to process a great deal of information (as here) to help them conceptualise the good, the detrimental impact of cognitive overload and fatigue on the actual valuation may well outweigh any (possibly small) advantages from removing such contextual effects. This is clearly an area for future research.

The same can be said for the potential inclusion of quantitative information on the underlying risk changes which would generate the shift in the 'ability to survive' curve, since there is some data to suggest that people have preferences over different risk distributions which generate the same life expectancy gain (Nielsen et al., 2010). As well as being factually more correct, it may help respondents understand better the increasing nature of the gain. Thus, a mixed methods approach may be most desirable in the future, one where respondents have access to both types of information to help them more fully understand the various, and complex, components of a life expectancy gain with a view to generating a VOLY that captures more, and not less, of these aspects. Another mixed-methods approach that may also be viable would be a two-stage procedure, such as that employed in Chilton et al. (2006), whereby a contextless value for a risk reduction is elicited, followed by a contextual one, the assumption being that any difference between the two would reflect the impact of the particular context in which the life expectancy gain was generated.

Turning to survey design, our study brings highlights the unintended but strong impact that the choice of wording can generate. In both studies described in the Introduction, the authors used the phrase "..due to slightly less wear and tear we are slightly healthier at each age than we would otherwise be and this feeds through to an increase in our life expectancy...". It is possible that the word 'slightly', while meant to imply insignificant, in fact (quite reasonably) may have signalled a noticeable impact on health quality, irrespective of any increase in longevity. In future given the pervasive influence of quality of life considerations, studies attempting to elicit values for life expectancy via an environmental improvement, should give attention to this in the design phase. Nevertheless, the pervasiveness of this attribute in respondents' 
thinking suggests a future need to develop an instrument that helps respondents disentangle the joint-product characteristics more effectively ${ }^{16}$. Alternatively, policymakers may have to accept that a VOLY could be biased due to this effect.

Additionally, respondents' quotes obtained from this qualitative study could be used in future studies to improve the information set by using language more familiar to the public, whilst still retaining its scientific validity. For example, instead of technical descriptions some colloquial phrases in Table 4 have the potential to be adapted to communicate continuous character of the gain, such as for example: "life extension through the whole life period", "prolonging the youth", "benefits through the years of my life", or "it would be stretched out over the time". Some of the other quotes such as: "Because it's not like that you need to survive 70 years to get 6 months more. It's possible to live only until 40 and then get proportionally less" or "I could get 1 day, in one year 2 days in the other and so on" could be a base to develop ways of better communicating the cumulative character of the life expectancy gain.

It is of course the case that the ultimate goal from improving respondent understanding is to obtain valid and consistent WTP measurement. Our sample was too small to conduct such an investigation directly, however, it may be recalled that a CVM study utilising this enhanced instrument generated data with a higher degree of consistency than an earlier comparator CVM study. The aim of this study was primarily to assess whether, after exposure to an enhanced information set, respondents were in a better position to value the good reliably than they otherwise would have been and, effectively, "bought what was "sold". The increased prominence given to the debriefing element of the CVM exercise has enabled us to assess this.

A potentially important finding for stated preference studies in general, which would also appear to support anecdotal evidence from previous studies, is that a contextualised instrument (primarily used to ground the study in reality for respondents) seems to encourage them to include irrelevant factors in their value construction, which we might term "baggage") but they would view as crucial attributes/outcomes of the policy. This implies that it is likely to be the case that at least some of the attributes described in a CVM information set - enhanced or otherwise - are likely to remain ill-defined as far as the respondent is concerned. It would be useful to know which ones, since this would help the researcher assess the costs to the reliability of the valuation, if only in relative terms (for example, non-consideration of one factor might be viewed as more serious than nonconsideration of another). Further, it would also be useful to identify which irrelevant factors remained in the valuation and an attempt made to find a reasonable proxy by which to control for this in the empirical analysis of the data.

\footnotetext{
${ }^{16}$ One possibility could be to extend the approach in Chilton et al. (2004) whereby respondents are first asked to value the bundle as a whole and then indicate their values for the component parts. These researchers confined themselves to the health benefits from air pollution reduction but it may be possible to include other benefits such as the environment or quality of life. However, very strong assumptions must be made in respect of underlying income and substitution effects for it to result in valid values.
} 


\section{References}

Alberini, A., Cropper, M., Krupnick, A., \& Simon, N. (2004). Does the Value of a Statistical Life vary with age and health status? Evidence from the US and Canada, Journal of Environmental Economics and Management, 48, 769-792.

Avitia, J., Costa-Font M., Gil J., \& Lusk J.L. (2011). A calibrate auction-conjoint experiment to elicit consumer valuation of sustainable farming: is agro-systems preservation relevant? Paper prepared for presentation at the EAAE 2011 Congress Change and Uncertainty

Baker, R. \& Robinson, A. (2004). Responses to Standard Gambles: are preferences 'wellconstructed'? Health Economics, 13, 37-48.

Blomquist G.C., Dickie M. \& O'Conor R.M. (2011). Willingness to pay for improving fatality risks and asthma symptoms: Values for children and adults of all ages, Resource and Energy Economics, 33(2), 410-425

Brunekreef, B., Miller B.G, \& Hurley J.F. (2007). The brave new world of lives sacrificed and saved, deaths attributed and avoided. Epidemiology, 18(6), 785-788.

Chilton, S.M., Covey, J., Jones-Lee, M., Loomes, G. \& Metcalf, H. (2004). Valuation of health benefits associated with reductions in air pollution: Final Report. DEFRA: London.

Chilton, S.M., \& Hutchinson, W.G. (1999). Do Focus Groups Contribute Anything to the Contingent Valuation Process? Journal of Economic Psychology, 20, 465-483

Chilton, S.M., Jones-Lee M., Kiraly, F., Metcalf, H. \& Pang, W. (2006). Dread risks. Journal of Risk and Uncertainty, 33, 165-182.

Chilton, S., Jones-Lee, M.W, Metcalf, H., \& Rabl, A. (2011). Communicating a gain in life expectancy from air pollution reduction in a Contingent Valuation study: An alternative approach. No. 2011-01 Newcastle Discussion papers in Economics ISSN 1361-1837.

Desaigues, B., Ami, D., Hutchison, M., Rabl, A., Chilton, S., Metcalf, H., Hunt.,A.,Ortiz, R. Navrud, S., Kaderjak, P., Szántó, R., Seested Nielsen, J., Jeanrenaud, C., Pellegrini, S., Braun Kohlová, M., Scasny, M., Vojtěch, M., Urban, J., Stoeckel, M-E., Bartczak, A., Markiewicz, O., Riera, P. \& Farreras, V. (2007). Final Report on the Monetray Valuation of Mortality and Morbidity Risks from Air Pollution. Framework VI Research Programme (Project no: 502687 'New Energy Externalities Developments for Sustainability' [NEEDS]).

Desaigues, B., Ami, D., Bartczak, A., Braun-Kohlová, M., Chilton, S., Czajkowski, M., Farreras, V., Hunt, A., Hutchison, M., Jeanrenaud, C., Kaderjak, P., Máca, V., Markiewicz, O., Markowska, A., Metcalf, H., Navrud, S., Seested Nielsen, J., Ortiz, R., Pellegrini, S., Rabl, A., Riera, P., Scasny, M., Stoeckel, M-E, Szántó, R., \& Urban, J. (2011). Economic valuation of air pollution mortality: a 9-country Contingent Valuation survey of Value of a Life Year (VOLY). Ecological Indicators. 11, 902910

Glaser, B. G., \& Strauss, A. L. (1967). The discovery of grounded theory: strategies for qualitative research. New York: Aldine

Hammitt, JK. (2007). Valuing changes in mortality risk: lives saved vs. life years saved. Review of Environmental Economics and Policy, Oxford University Press. 2007.

Jenkins, S. P. (2005). Survival Analysis, iser.essex.ac.uk 
Johannesson, M. \& Johansson, P-O. (1996). To be or not to be, that is the question: an empirical study of the WTP for an increased life expectancy at an advanced age. Journal of Risk and Uncertainty, 13, 163-174.

Johannesson, M. \& Johansson, P-O. (1997). Quality of life and the WTP for an increased life expectancy at an advanced age. Journal of Public Economics, 65, 219-228.

Krupnick, A., Cropper, M.L., Alberini A., Heintzelman, M.D., Simon N., O'Brien B. \& Goeree, R. (2000). Willingness to pay for improving fatality risks and asthma symptoms: Values for children and adults of all ages, Discussion Paper 00-37, Resources for the Future.

Krupnick, A., Alberini, A., Cropper, M., Simon, N., O’Brien, B., Goeree, R., \& Heintzelman, M. (2002). Age, health and the willingness to pay for mortality risk reductions: a contingent valuation study of Ontario residents. Journal of Risk and Uncertainty, 24, 161-186.

Mason, J. Qualitative Researching. London. Sage. 1996

Morris, J. \& Hammitt J. (2001). Using life expectancy to communicate benefits of health care programs in contingent valuation studies, Medical Decision Making, Nov-Dec., 468478.

Navrud, S., \& Ready, R. (2007). Eds, Reliability of benefit value transfers from contingent valuation data with forest-specific attributes in environmental value transfer: issues and methods. The Netherlands: Kluwer Academic Publishers.

Nielsen, J.S., Chilton, S.M., Metcalf, H., \& Jones-Lee, M.W. (2010). How would you like your gain in life expectancy to be delivered? An experimental approach, Journal of Risk and Uncertainty, 41(3), 195-218.

Nielsena J. B., Gyrd-Hansena D., Sønbø Kristiansena I., \& Nexøea, J. (2003). Impact of socio-demographic factors on willingness to pay for the reduction of a future health risk, Journal of Environmental Planning and Management, 46(1), 2003, 9-47

Payne, J.W., Bettman, J.R., \& Schkade, D.A. (1999). Measuring constructed preferences: towards a building code, Journal of Risk and Uncertainty, 19 (1-3), 243-270.

QSR NVivo Qualitative data analysis program (2007) Version 7. Melbourne, Australia, QSR International Pty Ltd.

Silverman D. (2001). Interpreting qualitative data: methods for analyzing talk, text and interaction. London: Sage.

Sundström K., \& H. Andersson (2009) Swedish consumers' wllingness to pay for food safety - a Contingent Valuation study on salmonella Rrsk, Working Papers from Swedish National Road \& Transport Research Institute (VTI) No 2009:2

\footnotetext{
Abbreviations:

$C V M=$ contingent valuation method,

CBA $=$ Cost-Benefit Analysis, $\mathbf{L E}=$ Life Expectancy, VOLY $=$ Value of a Life Year,

VSL $=$ Value of Statistical Life, WTP $=$ Willingness to Pay
} 


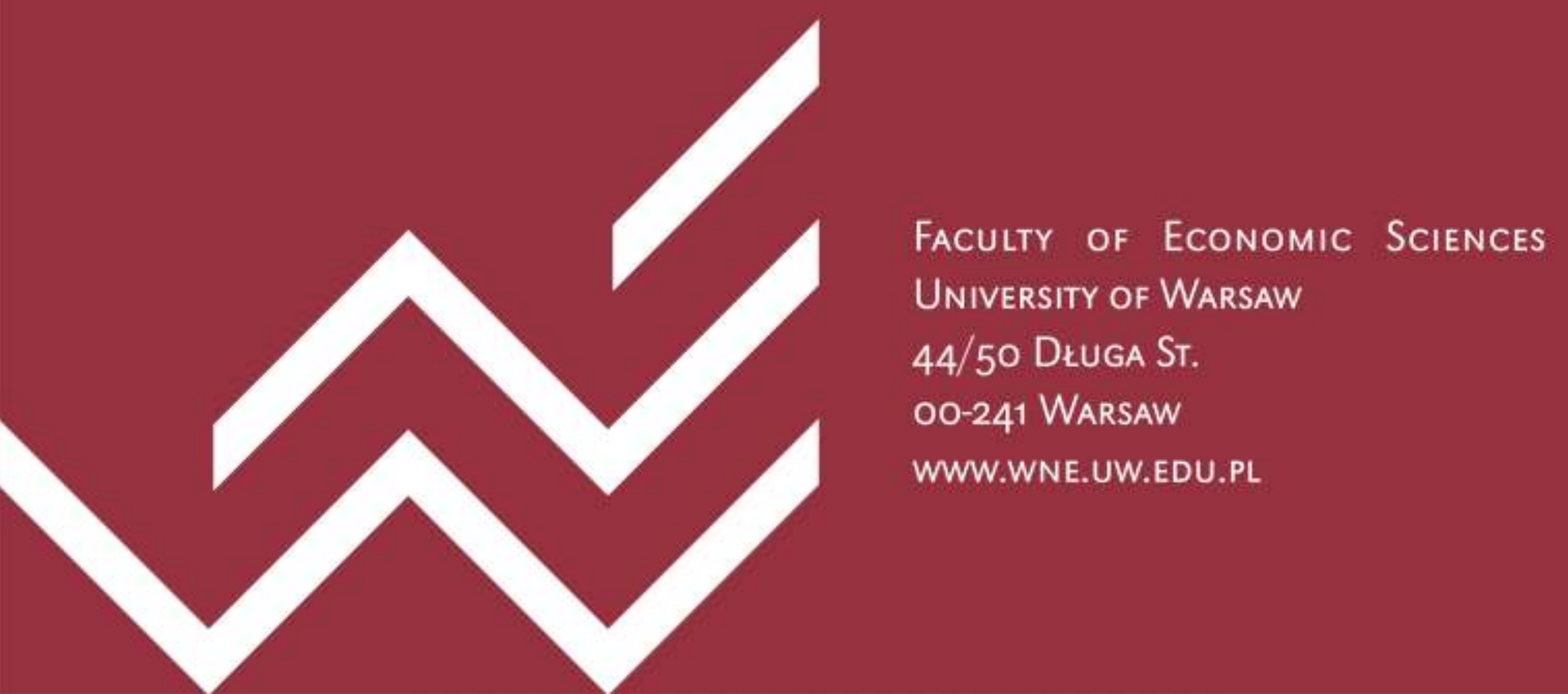

